\title{
Spontaneous intracranial hemorrhage as an initial manifestation of primary Sjögren's syndrome: a case report
}

\author{
Guo-Qiang Wang ${ }^{*+}$ and Wei-Wei Zhang ${ }^{\dagger}$
}

\begin{abstract}
Background: Sjögren's syndrome can involve the central nervous system; however, spontaneous intracranial hemorrhage has rarely been reported as the initial manifestation.

Case presentation: We report a 39-year-old woman with primary Sjögren's syndrome presenting with intracranial hemorrhage. The diagnosis of primary Sjögren's syndrome was based on the presence of ocular dryness, salivary gland secretory and excretory dysfunction confirmed with dynamic tracer emission CT, and positive anti-Sjögren's syndrome A and anti-Sjögren's syndrome B antibodies.

Conclusion: Primary Sjögren's syndrome can present with variable central nervous system signs, which may precede the classic sicca symptoms. Therefore, Sjögren's syndrome-associated indicators should be investigated in patients without the common risk factors for stroke who present with spontaneous intracranial hemorrhage.
\end{abstract}

Keywords: Sjögren's syndrome, Vasculitis, Intracranial hemorrhage, Internal carotid artery, Moyamoya disease, Anti-Sjögren's syndrome A antibody, Anti-Sjögren's syndrome B antibody

\section{Background}

Sjögren's syndrome (SS) is a common autoimmune disease. The histopathological hallmark is periductal lymphocytic infiltration of the exocrine glands, mainly the salivary and lacrimal glands, which results in loss of secretory function. The annual incidence has been estimated at 3.96.0 per 100,000 population $[1,2]$. SS has a marked female preponderance with a female-to-male ratio of 13:1. It also features a later age at onset, with a median age of 54 years for women and 58 years for men at the first diagnosis [3]. This syndrome is classified as primary SS (pSS) in the absence of other autoimmune diseases, and as secondary SS when it is associated with other autoimmune diseases such as rheumatoid arthritis, scleroderma, systemic lupus erythematosus, AIDS, hepatitis $\mathrm{C}$ infection, pre-existing lymphoma sarcoidosis, graft-versus-host disease, or the use of anticholinergic drugs.

The neurological manifestations of pSS involve both the peripheral and central nervous systems. Peripheral

\footnotetext{
*Correspondence: wgqbj@163.com

${ }^{\dagger}$ Equal contributors

Department of Neurology, General Hospital of Beijing Military Region, Beijing 100700, China
}

(c) 2013 Wang and Zhang; licensee BioMed Central Ltd. This is an Open Access article distributed under the terms of the

nervous system involvement is considered the most common neurological manifestation in pSS, and is characterized by predominantly sensory or occasionally mixed neuropathies, and mononeuritis multiplex. Central nervous system involvement occurs in $5.8-68 \%$ of pSS patients $[4,5]$ and, in most cases, neurological manifestations precede the sicca symptoms [6]. Central nervous system lesions in pSS vary from diffuse involvement, which manifests as cognitive deficits or meningoencephalitis, to focal involvement, which presents with similar symptoms to multiple sclerosis or neuromyelitis optica [7]. SS-associated infarction seldom occurs with stroke-like features such as aphasia or hemiplegia [5] and SS is even more rarely complicated with severe cerebral artery lesions, particularly hemorrhagic stroke. We report a case of a patient with pSS who presented with intracranial hemorrhage (ICH).

\section{Case presentation}

After suffering from acute headache for $2 \mathrm{~h}$ while washing clothes, a 39-year-old woman was admitted to the Department of Neurology, General Hospital of Beijing Military Region, in September 2012. The patient had no history of hypertension, coagulation disorders, or arthralgia, and 
showed no obvious xerostomia or xerophthalmia. On admission, her general examination was normal except for mild hypertension $(148 / 90 \mathrm{mmHg})$. The neurological examination revealed positive Kernig's sign only, without motor or sensory deficit. An urgent brain CT (Figure 1) showed hemorrhage in the left hippocampus, which extended into the ventricular system. Mannitol $250 \mathrm{~mL}$ was administered every $8 \mathrm{~h}$ for 1 week. Her headache gradually eased, the blood pressure returned to the normal range, and Kernig's sign disappeared.

All of the additional examination results were normal, including ambulatory blood pressure and electrocardiogram; chest CT; cardiac ultrasonography; and examinations of the digestive, urinary, and uterine systems, and the breasts and appendages. Vision in the left and right eyes was 0.6 and 0.5 , respectively. Schirmer's tear test results in both eyes were $<2 \mathrm{~mm}$ in $5 \mathrm{~min}$ (normal $>15 \mathrm{~mm}$ ); tear breakup time was $2 \mathrm{~s}$ (normal $\geq 10 \mathrm{~s}$ ); and punctate fluorescein staining was $>10$ (normal $<10)$.
The results of routine laboratory studies were also at normal levels, including complete blood cell count, coagulation, liver function, kidney and thyroid, lipids, glucose, glycosylated hemoglobin, C-reactive protein, and anti-O chain. Serological tests for HBsAg, hepatitis $C$ virus, human immunodeficiency virus, syphilis, and tumor markers were negative. Erythrocyte sedimentation rate (ESR) was $62 \mathrm{~mm} / 1 \mathrm{st}$ h; rheumatoid factor, $701 \mathrm{IU} / \mathrm{mL}$ (normal <25); antibody SS-A titer, $95 \mathrm{ng} / \mathrm{mL}$; SS-B, $58 \mathrm{ng} / \mathrm{mL}$; and recombinant Ro-52, $83 \mathrm{ng} / \mathrm{mL}$ (normal $<10$ ). Immunoglobulin (Ig) G was $18.7 \mathrm{~g} / \mathrm{L}$ (normal 6-16); IgA, $6.86 \mathrm{~g} / \mathrm{L}$ (normal, 0.4-3.3); IgM, $2.14 \mathrm{~g} / \mathrm{L}$ (normal, 0.4-2.6); complement C3, 0.44 g/L (normal, 0.85-1.93); and C4, $0.13 \mathrm{~g} / \mathrm{L}$ (normal, $0.12-0.36$ ). Other auto-antibodies, including anti- $\beta 2$-glycoprotein-I, anti-cardiolipin, anti-TB, and paraneoplastic antibody (anti-Hu, Yo, Ri, NMDA) were negative.

Brain MRI (Figure 2) performed 5 days after admission confirmed the diagnosis of hemorrhage in the left uncus
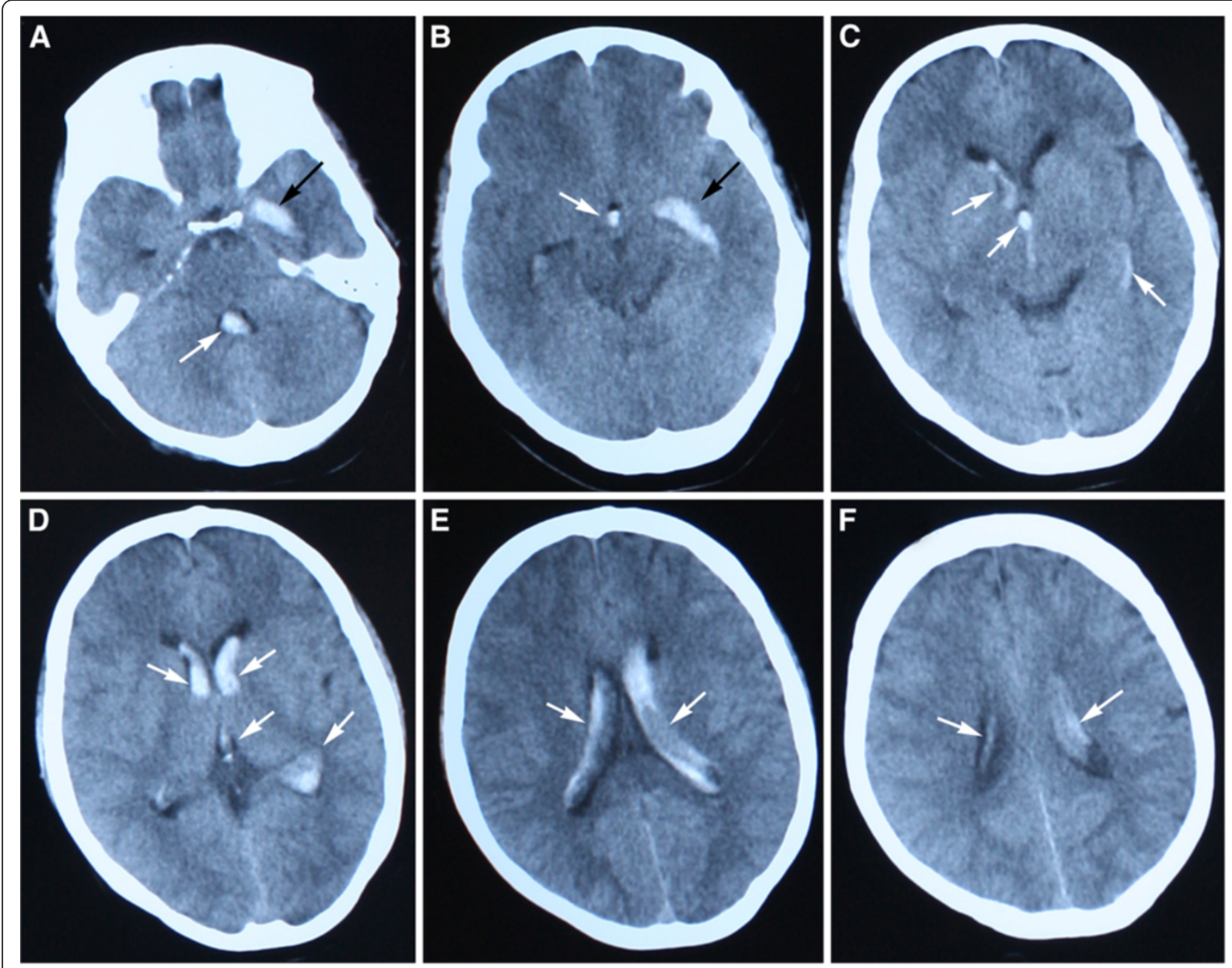

Figure 1 Brain CT obtained on the day of onset. The image shows hemorrhage in the left hippocampus (A, B, black arrow), which extends into the ventricular system (A-F, white arrows). 

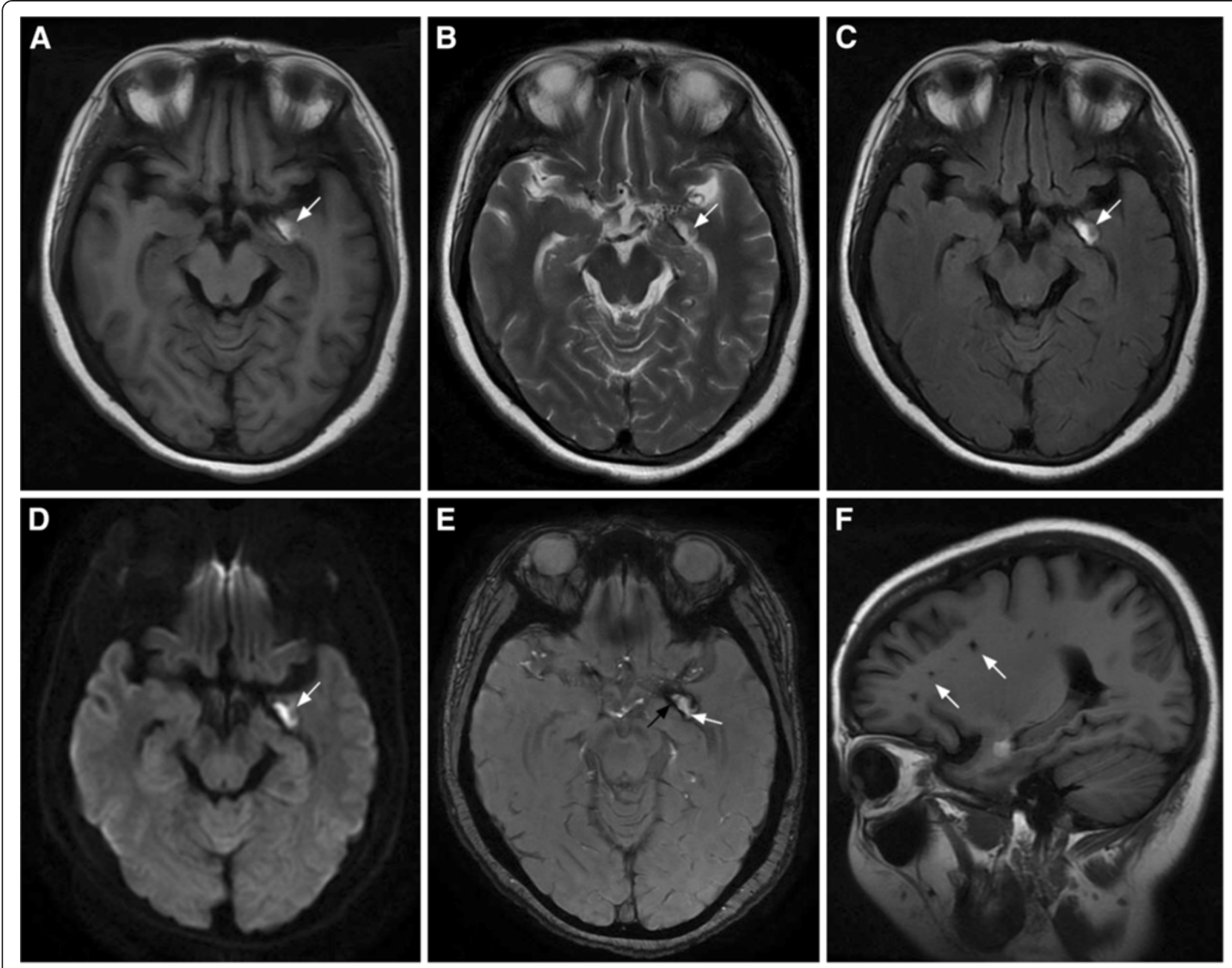

Figure 2 Brain MRI obtained 5 days post-onset. Axial T1, T2, T2-FLAIR, and DWI (A-D) show an abnormal high signal within the left lateral uncus (hippocampus) (arrows). On T2 star-weighted angiography imaging (E), the lesion shows markedly low signal intensity (black arrow) with a hyperintense core (white arrow). These findings indicate that the left hippocampal hemorrhage was subacute. Sagittal T1 (F) shows multiple lacunar low signals in the parietal and periventricular areas (arrows), suggesting multiple lacunar infarction in the cerebral hemispheres.

(hippocampus) with multiple lacunar infarcts in the cerebral hemispheres.

Cerebral angiography revealed occlusion of the terminal portion of the left internal carotid artery (Figure 3A and B). Mild collateral circulation from the right internal carotid artery (Figure $3 \mathrm{C}$ ) and the left posterior cerebral artery (Figure 3D) to the left middle cerebral artery region were present,and moyamoya vessels were slightly seen (Figure 3A, black arrow). No aneurysm, arteriovenous malformation or obvious vascular wall irregularity was found. The cerebral venous system was not involved.

Salivary gland dynamic tracer emission CT with 99mTc-sodium pertechnetate (Figure 4) showed decreased uptake in the left parotid and both submandibular glands, as well as reduced excretion in both sets of glands.
Based on the abnormal laboratory findings, the patient was diagnosed with pSS-associated cerebral vasculitis with ICH extending into the ventricular system, and with occlusion of the terminal portion of the left internal carotid artery accompanied by moyamoya vessels (quasi-moyamoya disease), as well as with multiple lacunar infarctions.

After the diagnosis, methylprednisolone $160 \mathrm{mg} /$ day was administered by intravenous injection for 1 week, with oral leflunomide $10 \mathrm{mg} /$ day. Follow-up CT was performed 2 weeks after admission and revealed that the hemorrhage was completely absorbed with no evidence of hydrocephalus; therefore the patient was discharged. At the 3-month follow-up, the patient was completely independent, with no discomfort, and normal physical examination findings. Laboratory tests showed positive results for SS-A/SS-B/Re-52 antibodies, and elevated 

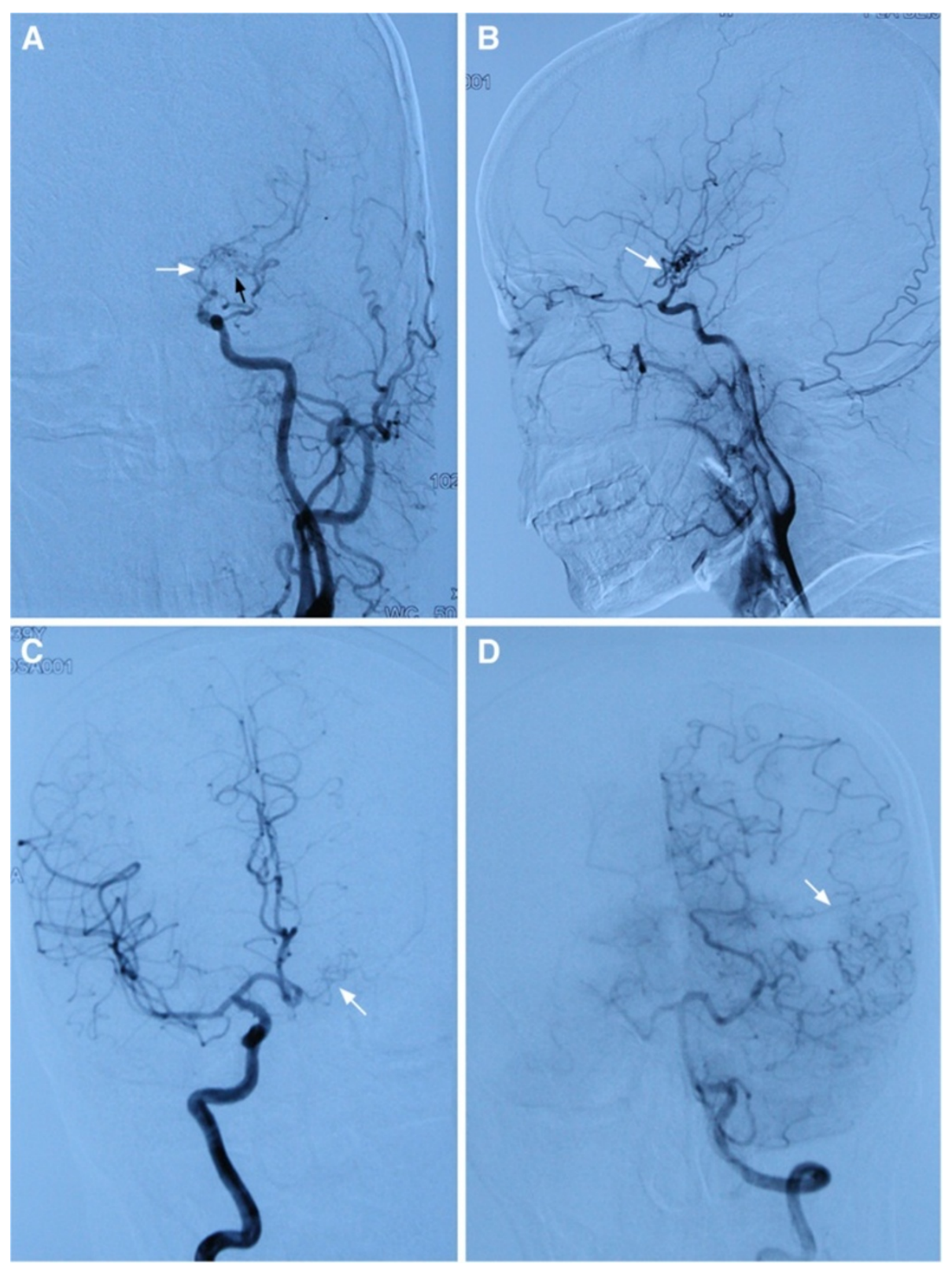

Figure 3 Cerebral angiography. The images show occlusion of the terminal portion of the left internal carotid artery (A, B, white arrow). Mild collateral circulation within the left middle cerebral artery region (C, D, white arrow) was also seen. The collateral circulation arose from the right internal carotid artery $(\mathbf{C})$ and the left posterior cerebral artery $(\mathbf{D})$ to the left middle cerebral artery region. A small number of moyamoya vessels can be seen (A, black arrow).

rheumatoid factor, but all of the titers were lower than that of the initial results.

\section{Conclusion}

Only five case of pSS-associated ICH have been reported [8-12]. Unlike our case, all of the five reported cases had a clear history of pSS prior to the ICH, and none had ICH as the first manifestation of pSS. Four patients developed aneurysms leading to subarachnoid hemorrhage [9-12].
Currently, vasculitis is a recognized feature of SS and is the pathological basis of the nervous system damage. Any vessels in the brain or spinal cord can be involved [5]. SSassociated systemic vasculitic manifestations occur in approximately $5-10 \%$ of SS patients [13] and two histopathological types have been suggested according to the type of the infiltrating cell: the neutrophilic type and the mononuclear cell type [14]. In SS-associated vasculitis, there is direct vessel wall invasion by neutrophilic or mononuclear cells, hyperplasia of endothelial and smooth 


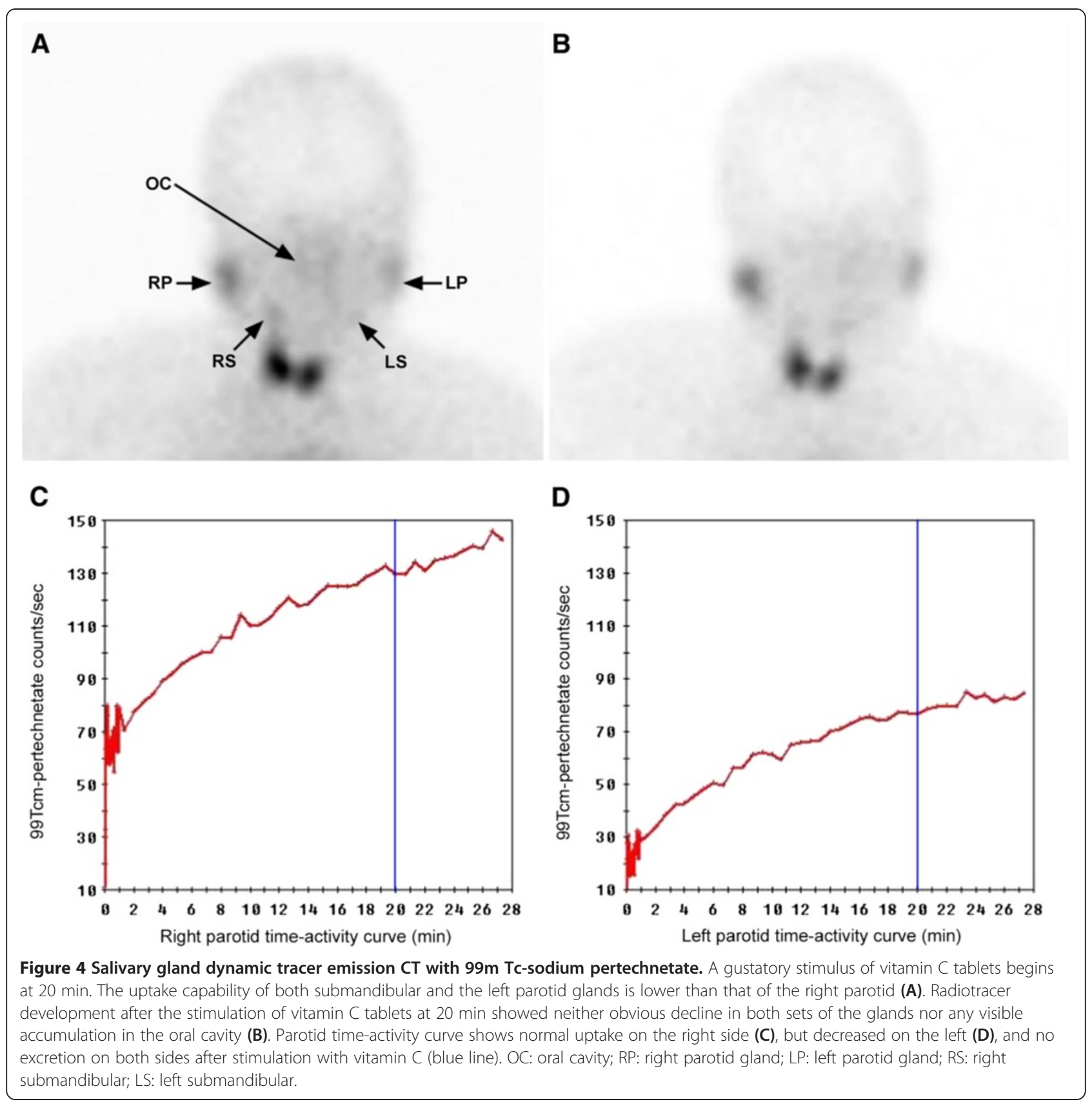

muscle cells, and immune complex deposits. These pathological factors alter or destroy the normal structure of the vessel wall, and result in vessel lumen occlusion, or wall rupture due to changes in local pressure at a weak point in the vessel wall [14]. Abnormal collateral vessels (moyamoya vessels) at the base of the brain may develop in autoimmune diseases such as SS $[8,15]$. In such conditions, the abnormal vascular network is termed quasi-moyamoya disease [16]. Moyamoya vessels are prone to thrombosis or hemorrhage due to fibrin deposits in their walls, fragmented elastic laminae, attenuated media, and microaneurysm formation [17].
It is well known that hemorrhagic stroke from moyamoya vessels occurs predominantly in adult moyamoya disease patients. If hypoperfusion is confirmed, bypass surgery may be considered to prevent rebleeding [16].

The most common serologic finding in SS is hypergammaglobulinemia, which is found in $80 \%$ of pSS patients [18]. The elevated immunoglobulin levels in SS patients contain a number of autoantibodies, including rheumatoid factors, and antinuclear, anti-SS-A and anti-SS-B antibodies [3]. The presence of these autoantibodies is closely related to the occurrence of vasculitis [14]. 
The non-specific and diverse nature of pSS leads to an average diagnostic delay of 3-8 years $[19,20]$, even though $100 \%$ of pSS patients have exocrine gland involvement. Patients presenting with sicca symptoms account for fewer than half of all pSS patients [20].

Our patient's sicca symptoms were not obvious clinically either before or after hemorrhage. Currently, the diagnosis of pSS is based on the following: high titers for anti-SS-A/Ro, SS-B/La, and recombinant Ro-52 antibodies and rheumatoid factor; elevated IgG and IgA; accelerated ESR; decreased exocrine glandular function; no trace of other autoimmune or infectious disease; and the revised classification criteria for Sjögren's syndrome proposed by the AmericanEuropean Consensus Group [21]. We hypothesized that in our patient, the pSS-associated vasculitis might have contributed to the development of the asymptomatic occlusion of the left terminal portion of the internal carotid artery and the subsequent development of moyamoya vessels, $\mathrm{ICH}$, and multiple lacunar infarctions.

Vasculitis-associated clues should be carefully investigated in adult female patients with stroke, particularly those without common stroke risk factors, such as hypertension or coagulopathy.

\section{Informed consent}

Written informed consent was obtained from the patient for publication of this Case report and any accompanying images. A copy of the written consent is available for review by the Editor of this journal.

\section{Competing interests}

We declare that we have no competing interests.

\section{Authors' contributions}

GQW and WWZ diagnosed and treated the patient, contributed equally to drafting and revising the manuscript. Both authors read and approved the final manuscript.

\section{Acknowledgements}

We wish to acknowledge Dr. Zhe Guo (Department of Nuclear Medicine, General Hospital of Beijing Military Region, China) for her technical support with radiological analyses and interpretation. We would like to thank Dr. RuJing Ma (Municipal People's Hospital, Qian'an, Hebei Province, China) and Dr. Yan-Xin Li (Department of Rheumatology and Immunology, General Hospital of Beijing, China) for their assistance in the patient' diagnosis and treatment. This work was supported by grant from the National Natural Science Foundation of China (No. 81070948).

Received: 28 February 2013 Accepted: 26 July 2013

Published: 29 July 2013

\section{References}

1. Plesivcnik Novljan M, Rozman B, Hocevar A, Grmek M, Kveder T, Tomsic M: Incidence of primary Sjogren's syndrome in Slovenia. Ann Rheum Dis 2004, 63:874-876.

2. Weng MY, Huang YT, Liu MF, Lu TH: Incidence and mortality of treated primary Sjogren's syndrome in Taiwan: a population-based study. J Rheumatol 2011, 38:706-708.
3. Goëb V, Salle V, Duhaut $P$, Jouen F, Smail A, Ducroix JP, Tron F, Le Loët X, Vittecoq O: Clinical significance of autoantibodies recognizing Sjögren's syndrome A (SS-A), SSB, calpastatin and alpha-fodrin in primary Sjögren's syndrome. Clin Exp Immunol 2007, 148:281-287.

4. Massara A, Bonazza S, Castellino G, Caniatti L, Trotta F, Borrelli M, Feggi L, Govoni M: Central nervous system involvement in Sjögren's syndrome: unusual, but not unremarkable clinical, serological characteristics and outcomes in a large cohort of Italian patients. Rheumatology (Oxford) 2010, 49:1540-1549.

5. Delalande S, de Seze J, Fauchais AL, Hachulla E, Stojkovic T, Ferriby D, Dubucquoi S, Pruvo JP, Vermersch P, Hatron PY: Neurologic manifestations in primary Sjögren syndrome: a study of 82 patients. Medicine (Baltimore) 2004, 83:280-291.

6. Mori K, lijima M, Koike H, Hattori N, Tanaka F, Watanabe H, Katsuno M, Fujita A, Aiba I, Ogata A, Saito T, Asakura K, Yoshida M, Hirayama M, Sobue G: The wide spectrum of clinical manifestations in Sjögren's syndromeassociated neuropathy. Brain 2005, 128:2518-2534.

7. Fauchais AL, Magy L, Vidal E: Central and peripheral neurological complications of primary Sjögren's syndrome. Presse Med 2012, 41:e485-e493.

8. Asai Y, Nakayasu H, Fusayasu E, Nakashima K: Moyamoya Disease Presenting as Thalamic Hemorrhage in A Patient With Neuromyelitis Optica and Sjögren's Syndrome. J Stroke Cerebrovasc Dis 2012, 21:619. e7-9.

9. Ishizaka S, Hayashi K, Otsuka M, Fukuda S, Tsunoda K, Ushijima R, Kitagawa N, Suyama K, Nagata I: Syringomyelia and arachnoid cysts associated with spinal arachnoiditis following subarachnoid hemorrhage. Neurol Med Chir (Tokyo) 2012, 52:686-690.

10. Hayashi K, Morofuji Y, Suyama K, Nagata I: Recurrence of subarachnoid hemorrhage due to the rupture of cerebral aneurysms in a patient with Sjögren's syndrome: case report. Neurol Med Chir (Tokyo) 2010, 50:658-661.

11. Klingler JH, Gläsker S, Shah MJ, Van Velthoven V: Rupture of a spinal artery aneurysm attributable to exacerbated Sjögren syndrome: case report. Neurosurgery 2009, 64:e1010-e1011.

12. Giordano MJ, Commins D, Silbergeld DL: Sjögren's cerebritis complicated by subarachnoid hemorrhage and bilateral superior cerebellar artery occlusion: case report. Surg Neurol 1995, 43:48-51.

13. Feist $E$, Hermann $K G$, Dankof A: Vasculopathy in Sjögren's syndrome. Z Rheumatol 2009, 68:305-311.

14. Molina R, Provost TT, Alexander EL: Two types of inflammatory vascular disease in Sjögren's syndrome. Differential association with seroreactivity to rheumatoid factor and antibodies to Ro (SS-A) and with hypocomplementemia. Arthritis Rheum 1985, 28:1251-1258.

15. Yanagawa $Y$, Sugiura T, Suzuki K, Okada Y: Moyamoya disease associated with positive findings for rheumatoid factor and myeloperoxidase-anti -neutrophil cytoplasmic antibody. West Indian Med J 2007, 56:282-284.

16. Hayashi K, Horie N, Suyama K, Nagata I: An epidemiological survey of moyamoya disease, unilateral moyamoya disease and quasi-moyamoya disease in Japan. Clin Neurol Neurosurg 2013, 115:930-933.

17. Yamashita M, Oka K, Tanaka K: Histopathology of the brain vascular network in moyamoya disease. Stroke 1983, 14:50-58.

18. Kassan SS, Moutsopoulos HM: Clinical manifestations and early diagnosis of Sjögren syndrome. Arch Intern Med 2004, 164:1275-1284.

19. Pavlidis NA, Karsh J, Moutsopoulos HM: The clinical picture of primary Sjögren's syndrome: a retrospective study. J Rheumatol 1982, 9:685-690.

20. Markusse HM, Oudkerk M, Vroom TM, Breedveld FC: Primary Sjögren's syndrome: clinical spectrum and mode of presentation based on an analysis of 50 patients selected from a department of rheumatology. Neth J Med 1992, 40:125-134.

21. Vitali C, Bombardieri S, Jonsson R, Moutsopoulos HM, Alexander EL, Carsons SE, Daniels TE, Fox PC, Fox Rl, Kassan SS, Pillemer SR, Talal N, Weisman MH, European Study Group on Classification Criteria for Sjögren's Syndrome: Classification criteria for Sjögren's syndrome: a revised version of the European criteria proposed by the American-European Consensus Group. Ann Rheum Dis 2002, 61:554-558.

doi:10.1186/1471-2377-13-100

Cite this article as: Wang and Zhang: Spontaneous intracranial hemorrhage as an initial manifestation of primary Sjögren's syndrome: a case report. BMC Neurology 2013 13:100. 